\title{
ERRATA
}

\section{Erratum: Touching Base}

Nat. Genet. 36, 327 (2004).

The Executive Director of the IGC expO project (http://www.intgen.org) is Michael Berens.

\section{Erratum: A previously unidentified MECP2 open reading frame defines a} new protein isoform relevant to Rett syndrome

G N Mnatzakanian, H Lohi, I Munteanu, S E Alfred, T Yamada, P J M MacLeod, J R Jones, S W Scherer, N C Schanen, M J Friez, J B Vincent \& B A Minassian Nat. Genet. 36, 339-341 (2004).

In the bottom half of Figure 1b, the second EST is a mouse Mecp2B EST (BU517697; IMAGE 6515311). 